\title{
Dissociating Barrel Development and Lesion-Induced Plasticity in the Mouse Somatosensory Cortex
}

\author{
Alexandra Rebsam, ${ }^{1}$ Isabelle Seif, ${ }^{2}$ and Patricia Gaspar ${ }^{1}$ \\ ${ }^{1}$ Institut National de la Santé et de la Recherche Médicale U616, Université Paris VI Hôpital Salpêtrière, 75651 Paris cedex 13, France, and ${ }^{2}$ Faculté de \\ Pharmacie, Université de Paris-Sud, 92296 Chātenay-Malabry, France
}

In the mouse somatosensory cortex, thalamocortical axons (TCAs) corresponding to individual whiskers cluster into restricted barrel domains during the first days of life. If whiskers are lesioned before that time, the cortical space devoted to the afferents from the damaged whisker shrinks and becomes occupied by thalamocortical afferents from neighboring unlesioned whiskers. This plasticity ends by postnatal day 3 (P3) to $\mathrm{P} 4$ when barrels emerge. To test whether TCA development and lesion-induced plasticity are linked, we used monoamine oxidase A knock-out (MAOA-KO) mice in which normal TCA development is halted by an excess of serotonin. Normal TCA development can be restored when serotonin levels are lowered by parachlorophenylalanine (PCPA). By varying the time of PCPA administration, we found that barrel development can be reinitiated until P11, although the emergence of TCA clusters becomes gradually slower and less complete. In mice in which barrels emerge $3 \mathrm{~d}$ later than the normal schedule, at P6 instead of P3, we examined lesion-induced plasticity. We find a progressive decline of the lesion-induced plasticity and a closure at P3, similar to normal mice, showing that this plasticity is not influenced by an excess of serotonin levels. Thus, in MAOA-KO mice, the emergence of barrel patterning can be delayed without a concomitant delay in lesion-induced plasticity, and the cortical space devoted to one whisker representation cannot be modified by the periphery once patterning is imprinted in the subcortical relays. We conclude that the closure of the lesioninduced plasticity period in the barrelfield is probably not determined at the cortical level.

Key words: lesion; whisker; serotonin; barrelfield; thalamocortical; monoamine oxidase A

\section{Introduction}

The somatosensory cortex of rodents is a useful model for analyzing the influence of sensory periphery and neural activity on pattern formation. In the cerebral cortex, each barrel corresponds to one whisker on the contralateral snout (Woolsey and Van der Loos, 1970). A barrel is formed by a bouquet of thalamocortical axon (TCA) terminals (Killackey and Leshin, 1975) around which layer IV stellate neurons aggregate (Woolsey et al., 1975). The clustering of TCAs into whisker-related patterns emerges during the first 3 postnatal days from an initially diffuse distribution in layer IV (Senft and Woolsey, 1991; Rebsam et al., 2002). During this period, the somatosensory map is plastic to changes induced by peripheral lesions (Van der Loos and Woolsey, 1973): lesioning a whisker row causes a reduction in the representation of this deprived row, whereas the representation of the adjacent rows expands. This effect diminishes with age, and no effect is visible when lesions are effected at postnatal day 4 (P4), at a time when thalamocortical fibers are clearly segregated into barrel domains (Woolsey and Wann, 1976; Belford and Kil-

Received 0ct. 8, 2004; revised Nov. 24, 2004; accepted Nov. 29, 2004.

This work was supported by Institut National de la Santé et de la Recherche Médicale, Centre National de la Recherche Scientifique, and Fondation de France. A.R. is funded by a grant from Fondation France Télécom Mécénat Autisme. We thank Nicole Ropert, Aude Muzerelle, and Constantino Sotelo for their help.

Correspondence should be addressed to Patricia Gaspar, Institut National de la Santé et de la Recherche Médicale U616, Pavillon Enfant et Adolescent, Centre Hospitalier Universitaire Pitié-Salpêtrière, 47 Boulevard de I'Hopital, 75013 Paris, France. E-mail: gaspar@infobiogen.fr.

DOI:10.1523/JNEUROSCI.4191-04.2005

Copyright $\odot 2005$ Society for Neuroscience $\quad$ 0270-6474/05/250706-05\$15.00/0 lackey, 1980; Jeanmonod et al., 1981). Thus, the maturation of the barrelfield and its plasticity are concomitant, suggesting that the two processes are linked (Woolsey and Wann, 1976). To test this hypothesis, a number of authors have tried to uncouple these phenomena (Vongdokmai, 1980; Osterheld-Haas et al., 1994). However, these results can be questioned because the delay in barrel emergence was limited and the experimental paradigms that were used implied a general delay of brain maturation. To test whether these developmental events are linked, we chose a model that allowed to delay the formation of barrels without affecting the general brain maturation. We used the monoamine oxidase A knock-out (MAOA-KO) mouse, in which barrels are lacking in the cerebral cortex despite a normal patterning of the subcortical sensory relays. The altered segregation of thalamocortical axons is attributable to an excess buildup of serotonin (5-HT) in the brain (Cases et al., 1996). The MAOA-KO mice can be made to develop a normal barrelfield when the $5-\mathrm{HT}_{1 \mathrm{~B}}$ receptors are genetically invalidated (Salichon et al., 2001; Rebsam et al., 2002) or when 5-HT levels are reduced by daily injections of parachlorophenylalanine (PCPA) from birth to P6 (Cases et al., 1996). PCPA is an inhibitor of tryptophan hydroxylase that significantly lowers 5-HT levels after $24-48 \mathrm{~h}$. We delayed the administration of PCPA to determine until when barrels can be induced to form in the MAOA-KO mice and found that this can occur until P10. Then, using mice with a $3 \mathrm{~d}$ delay in barrel emergence, we analyzed the effects of peripheral lesions on barrel development. 


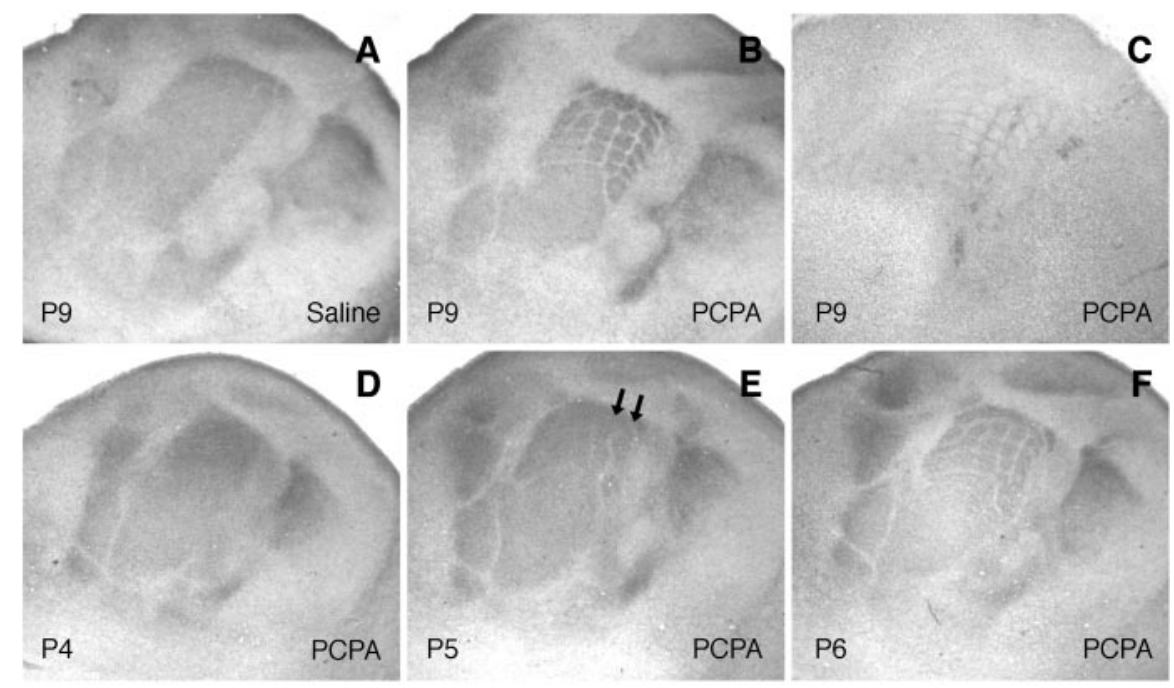

Figure 1. Rescue of large barrels in MAOA-KO mice treated with PCPA. Tangential sections of flattened hemispheres of MAOA-KO mice are shown. Mice were treated with saline $(A)$ or PCPA $(B-F)$ treatment from P2.5-HTT immunohistochemistry $(A$, $B, D-F)$ reveals the sensory thalamocortical fibers and Nissl staining $(C)$ in the layer IV neurons. $A-C$, A rescue of barrel clustering in the large whisker representation is noted at P9 in PCPA-treated mice (from $P 2$ to $P 8 ; B, C$ ) but not in the saline-treated mice $(A)$. $D-F$, In MAOA-K0 mice treated from $P 2$ on, no segregation of fibers is visible at $P 4(D)$, rows $A$ and $B$ (arrows) corresponding to the large whiskers are visible at $\mathrm{P} 5(E)$, and individual barrels are observed at $\mathrm{P} 6(F)$.

\section{Materials and Methods}

Animals. Wild-type mice of the $\mathrm{C} 3 \mathrm{H} / \mathrm{HeOuJ}$ strain were purchased from a commercial source (Iffa Credo, Arbresle, France). The MAOA-KO mouse line has been described by Cases et al. (1995). Pregnant mice of each strain were examined twice daily to determine the moment of birth (noted as $\mathrm{P} 0$ ) with a $12 \mathrm{~h}$ precision.

All of the experimental procedures were performed in accordance with the provision for animal care and use of the European Community Council.

PCPA treatment. P2-P21 pups were injected with PCPA (300 mg/kg, s.c., in saline) every $24 \mathrm{~h}$ for 1 week. The litter was separated from the mother, and all of the pups received PCPA or saline and were returned together to home cages.

HPLC. Pups from MAOA-KO mice were decapitated at P2, P3, P4, P5, P6, P7, P9, or P11, after a single PCPA or saline injection $(300 \mathrm{mg} / \mathrm{kg}$, s.c.) and also after daily injections at different time points after the treatment (from 6 to $118 \mathrm{~h}$ ). Brains were dissected, weighted, and kept at $-80^{\circ} \mathrm{C}$. Quantification of monoamines by reverse-phase paired-ion HPLC was as described by Salichon et al. (2001), with some minor modifications.

Row C lesion. P0-P5 neonatal littermate mice were anesthetized by hypothermia, the central $C$ row of whisker follicles was electrocauterized, and the damaged follicles were removed with forceps. The pups were allowed to recover before being returned to their home cages. Lesioned mice were killed at P9. The whisker pads were sectioned tangentially on a cryostat and stained with Nissl to check that the lesions were effective.

5-HTT immunohistochemistry, cytochrome oxidase cytochemistry, and Nissl staining. Eighty-one mice [C3H $(n=22)$ or MAOA-KO $(n=59)$ ] from P9 to P31 were anesthetized with $1 \%$ sodium pentobarbital (200 $\mathrm{mg} / \mathrm{kg}$, i.p.) and perfused through the aorta with $4 \%$ paraformaldehyde in $0.12 \mathrm{M}$ phosphate buffer $(\mathrm{PB})$. The cerebral hemispheres were separated and flattened between two glass slides with spacers and postfixed overnight in the same fixative. After cryoprotection in PB with $30 \%$ sucrose, tangential sections of the flattened hemispheres were cut to 50 $\mu \mathrm{m}$-thick sections on a freezing microtome and processed for 5-HT transporter (5-HTT) immunohistochemistry, as described by Rebsam et al. (2002), and for cytochrome oxidase (CO) cytochemistry or Nissl staining, as described by Salichon et al. (2001).

Quantitative analysis. 5-HTT-immunostained and CO-stained sections were visualized under a light microscope, and images were acquired using a CoolSNAP digital camera (Photometrics, Tucson, AZ). Measurements of the entire large barrels area and surface areas devoted to each row (C to D) were made using MetaMorph software (Universal Imaging, Downington, PA). Barrel septa were included in the row measurements. For each hemisphere, areas C and D were normalized for the posteromedial barrel subfield (PMBSF) area. The $\mathrm{D} / \mathrm{C}$ ratio was used as a plasticity index (Schlaggar et al., 1993). Additional qualitative evaluations of the plasticity were done by measuring the degree of fusion in barrel row $\mathrm{C}$. The sections were coded, and, one investigator, blind to the experimental condition of the cases, assigned them to three categories (I, total fusion of row $\mathrm{C}$; II, partial fusion of row $\mathrm{C}$; III, no fusion of row $\mathrm{C}$ ).

\section{Results}

Recovery of barrel patterning in MAOAKO mice by late PCPA injection

The effect of PCPA on brain levels of 5-HT in the MAOA-KO mice was analyzed. After a single PCPA injection at P2, we found significant reductions of 5 -HT by $21 \%$ after $24 \mathrm{~h}(175 \pm 4$ vs $220 \pm 1 \mathrm{ng} ; n=6$ vs $n=4 ; p<0.0001 ; t$ test), by $34 \%$ at $48 \mathrm{~h}$ $(242 \pm 10 \mathrm{ng}$ vs $368 \pm 4 \mathrm{ng} ; n=6$ vs $n=4 ;$ $p<0.0001)$, and by $39 \%$ at $65 \mathrm{~h}(247 \pm 19$ ng vs $404.75 \pm 5.19$ ng; $n=2$ vs $n=4 ; p<0.0004)$. Repeated PCPA injections caused more substantial decreases of 5-HT (i.e., three consecutive PCPA injections at P2, P3, and P4 caused a 47\% decrease $65 \mathrm{~h}$ after the first injection).

To assess the formation of periphery-related patterns, we used 5-HTT immunostaining that labels sensory thalamocortical axons until P12 (Lebrand et al., 1996). We also used cytochrome oxidase staining that labels both thalamic and cortical components of the barrels (Wong-Riley and Welt, 1980). Untreated or saline-treated MAOA-KO mice display no visible barrel patterning (Cases et al., 1996) (Fig. 1A). Daily treatments with PCPA from P2 to P8 $(n=16)$ rescued TCA patterning in the PMBSF (Fig. $1 B$ ). Nissl staining showed a barrel-like aggregation of layer IV neurons in this area (Fig. $1 C$ ), indicating that the maturation of the barrel structure is complete. Barrel emergence was also observed when PCPA treatments were started between P3 and $\mathrm{P} 10$, and patterning was examined with 5-HTT immunohistochemistry before $\mathrm{P} 12$ or with $\mathrm{CO}$ at $\mathrm{P} 21$ and $\mathrm{P} 28$ (Fig. $2 A, B)(n=$ 28). No barrels were observed when PCPA was started at P15 (Fig. $2 C)(n=3)$ or P21 $(n=3)$, and CO patterns were examined, respectively, at $\mathrm{P} 28$ and $\mathrm{P} 31$.

The barrel patterning that is obtained in the MAOA-KO mice by late PCPA treatments is partial because it concerns only the larger barrels in the PMBSF corresponding to the large snout whiskers. This is in contrast to early PCPA treatments starting on the day of birth, which lead to the rescue of barrels in the entire barrelfield, including the small anterior snout vibrissas (Cases et al., 1996). When PCPA treatment was delayed further, at P8 or $\mathrm{P} 10$, the recovery tended to become limited to some barrel rows and the outline of barrels became progressively less clear (Fig. $2 A, B)$. These results indicate that TCAs have the capacity to remodel within layer IV in the cerebral cortex, allowing the emergence of separate periphery-related patterns, but this capacity becomes gradually reduced over the first 2 postnatal weeks. 


\section{Timing of TC fiber segregation in PCPA-treated MAOA-KO mice}

In normal mice and in $\mathrm{MAOA} / 5-\mathrm{HT}_{1 \mathrm{~B}}$ double $\mathrm{KO}$ mice, barrel emergence follows a clearly defined topographic sequence, with progressive separation of body representations at P1, emergence of whisker rows in the PMBSF at P2, and individualization of barrels by P3 (Rebsam et al., 2002). We followed TC segregation after administration of PCPA at P2 in MAOA-KO littermate mice. One pup was killed every day and examined by 5 -HTT immunocytochemistry on tangential sections. At P3, the TCAs remained uniformly distributed (data not shown); a separation between the lower lip and the anterior snout representations was visible at P4 (Fig. 1D). At P5, the limits among the lower lip, the forepaw, and the hindpaw representations emerged and whisker rows became visible (Fig. 1E). At P6, TCA clusters corresponding to individual whiskers began to be detectable (Fig. $1 F$ ). Thus, the developmental sequence is similar to that observed in normal development but is delayed by $3 \mathrm{~d}$.

This developmental sequence appeared to be protracted when PCPA was started a few days later, at P5: $4 \mathrm{~d}$ after PCPA administration, at $\mathrm{P} 9$, the thalamocortical axons formed row-like arrangements and clustered into barrels within these rows at P12, $7 \mathrm{~d}$ after the beginning of drug administration (data not shown).

\section{Effect of row C lesion on MAOA-KO mice rescued by PCPA injection}

We analyzed the lesion-induced plasticity in MAOA-KO littermate mice that were administered PCPA from P2 to P8, because this protocol was the one that provided the most reliable recovery of the large barrels in the PMBSF. As controls, we used wild-type mice of the inbred $\mathrm{C} 3 \mathrm{H} / \mathrm{HeOuJ}$ strain. Electrocauterization of whisker row $\mathrm{C}$ at $\mathrm{P} 1$ (Fig. $3 A, D$ ) resulted in the fusion of the corresponding cortical barrels (Table 1 ) and a decrease in the area of row $\mathrm{C}$, whereas adjacent rows (rows $\mathrm{B}$ and $\mathrm{D}$ ) became enlarged (Fig. $3 A, D$ ) in both genotypes. The effects of this lesion decreased rapidly at later ages (Fig. $3 B, E$ ) and ceased when lesions were made at P4 (Woolsey and Wann, 1976; Jeanmonod et al., 1981) (Fig. 3C,F). A similar decline in the plasticity responses was observed qualitatively (Fig. 3A-F, Table 1) and quantitatively (Fig. $3 G-I)$. Statistical analyses of rows $\mathrm{C}$ and $\mathrm{D}$ normalized areas (Fig. $3 G, H$, respectively), as well as $\mathrm{D} / \mathrm{C}$ ratio (Fig. $3 I$ ), showed no significant differences between the MAOA-KO rescued and the control mice at all of the ages examined with the ANOVA test $(p<0.05)$. Thus, the critical period for lesion-induced plasticity closes at the same time (P3) in the treated MAOA-KO mice, and the closure of the lesion-induced plasticity period is not related to the timing of whisker row and barrel development.

\section{Discussion}

The present study demonstrates that lesion-induced plasticity in the barrelfield is not dependent on the time of emergence of thalamocortical patterning in the cerebral cortex. Thalamocortical segregation into whisker rows and into barrels can be significantly delayed, whereas sensory deprivation produces its effects only during a limited time period.

Thalamic afferents to the primary somatosensory cortex have been shown to be diffusely distributed in layer IV of the cerebral cortex before they cluster into columnar domains that correspond to the sensory afferents of a given whisker (Senft and
Woolsey, 1991; Rebsam et al., 2002). This segregation begins by P2-P3 and involves important axon remodeling with the formation and elimination of axon branches. This process is severely disrupted in the MAOA-KO mice, which have a 50\% reduction in the number of TCA collaterals, as well as an increase in the lateral spread of the thalamocortical axon arbors within layer IV at P7 (Rebsam et al., 2002). The present study shows that, when 5-HT levels are lowered between P4 and P11, a periphery-related clustering of the diffusely distributed thalamocortical axons can still occur, indicating that thalamocortical axon arbors remain plastic during this period. These findings imply that TCAs are still able to form new collaterals and retract exuberant branches to make barrel patterns emerge. A protracted period of thalamocortical segregation was also indicated in rats in which a MAOA-KO-like phenotype was induced by administering the MAOA inhibitor clorgyline, because barrel development was resumed after discontinuing clorgyline treatment at P6 (Boylan et al., 2001). In the present study, this capacity of TCAs to remodel becomes progressively reduced over time. Indeed, there is a lack of rescue of the smaller anterior snout barrels with late PCPA treatment, and a fewer barrels become rescued as PCPA treatment is delayed further. The loss of the ability of thalamocortical axons to segregate beyond P11 could be related to the loss of some intrinsic developmental characteristics of thalamic neurons after this period. A large number of genes, potentially involved in synaptic plasticity and axon growth, have been found to be transiently expressed in the ventrobasal thalamic nuclei, with a time course that could match the time course of this form of TCA plasticity because they are maximal during the first postnatal week, with a rapid decrease of expression after P10. Among these genes, the kainate receptors (Kidd et al., 2002), adenylate cyclase 1 (Nicol et al., 2005), the serotonin transporter, and the $5-\mathrm{HT}_{1 \mathrm{~B}}$ receptor (for review, see Gaspar et al., 2003) could potentially be important for permitting this developmental remodeling of thalamocortical axons to occur.

Interestingly, although TCA remodeling can be significantly delayed, the influence of the periphery in this process seems to be negligible beyond P3. Indeed, the steady decline of lesioninduced plasticity and its complete closure at P3 is remarkably similar when cortical patterns emerge at P2-P3 or $3 \mathrm{~d}$ later. These results differ from previous experiments in which barrel development was delayed by 1 or $2 \mathrm{~d}$ and in which the lesion-induced plasticity was correspondingly delayed (Vongdokmai, 1980). In this previous model, however, a general growth retardation could affect the development of all of the sensory relays. In contrast, the MAOA-KO mice do not display growth deficits, whether or not they are treated with PCPA, and have been shown to develop normal trigeminal patterning in the brainstem and thalamus (Cases et al., 1996). Thus, the independence of TCA rearrange- 

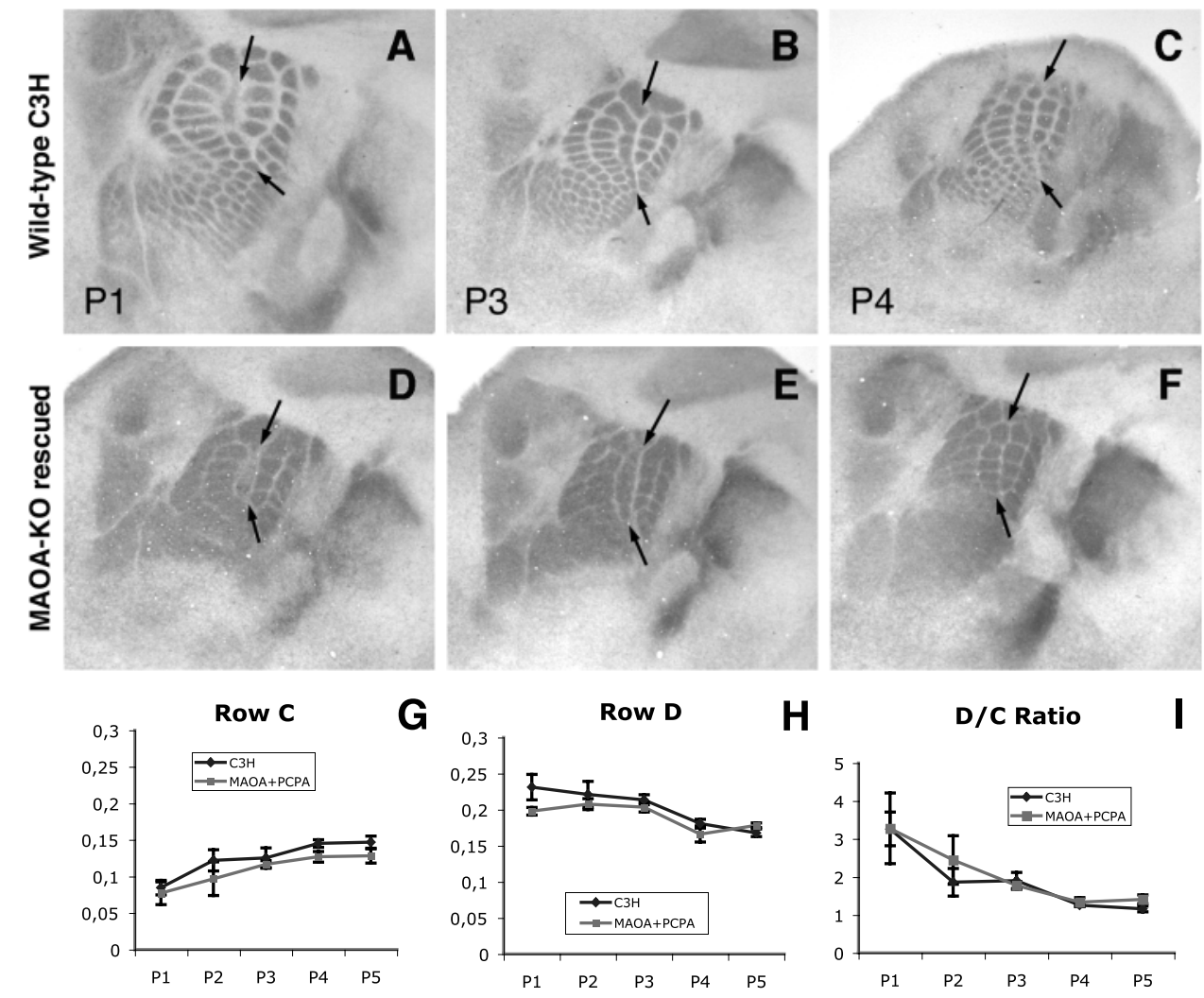

Figure 3. Critical period for lesion-induced plasticity is unchanged in MAOA-KO rescued mice. Quantification of the row Clesion effect is shown. $A-F$, Row $C$ whiskers were electrocauterized at $P 1$ $(A, D), P 2, P 3(B, E), P 4(C, F)$, and $\mathrm{P} 5$ in $C 3 \mathrm{H}$ mice $(A-C ; n=40)$ and MAOA-KO rescued mice $(D-F ; n=34) . A, D$, Lesions at $\mathrm{P} 1$ cause a decrease of row $C$ and an expansion of adjacent barrel rows $B$ and $D$ cortical areas in both $C 3 H(A)$ and MAOA-KO rescued $(D)$ mice. $B$, E, The lesion still has a milder effect at $P 3$. $C, F$, No effect is visible when row $C$ lesion is made at $P 4$ and assessed with 5 -HTT immunolabeling. $G-I$, Quantification of lesion effect. The areas of rows $C(G)$ and $D(H)$ were compared with the total area of the large barrels for normalization. $A$, At P1, the row $C$ area is low and increases progressively with age until P4 in MAOA-KO rescued and normal mice. $B$, Conversely, the row D area decreases with age from P1 to P4 in wild-type mice. A slight difference can be observed because the row D area seems grossly identical from P1 to P3 in MAOA-K0 rescued mice. A decrease is clearly observed between P1 and P4, as well as stabilization in relative area size from P4 to P5 in both genotypes. The $\mathrm{D} / \mathrm{C}$ ratio plasticity index clearly indicates a general decrease from $\mathrm{P} 1$ to $\mathrm{P} 4$ and stabilization thereafter, stating the absence of plasticity after $\mathrm{P3}$. $\mathrm{C} 3 \mathrm{H}$ mice values are in black, and MAOA-KO rescued mice values are in gray. Arrows indicate row C. Error bars represent SEM.

Table 1. Effects of whisker lesions of row C on TCA clustering in the cortex

\begin{tabular}{lllcr}
\hline Age at lesion & Genotype & Class I & Class II & Class III \\
\hline P0-P1 & C3H $(n=9)$ & $89 \%$ & $11 \%$ & $0 \%$ \\
& MAOA-KO $(n=8)$ & $88 \%$ & $13 \%$ & $0 \%$ \\
P2-P3 & C3H $(n=13)$ & $46 \%$ & $23 \%$ & $31 \%$ \\
& MAOA-KO $(n=14)$ & $31 \%$ & $46 \%$ & $23 \%$ \\
P4-P5 & C3H $(n=19)$ & $0 \%$ & $0 \%$ & $100 \%$ \\
& MAOA-KO $(n=13)$ & $0 \%$ & $0 \%$ & $100 \%$
\end{tabular}

Mice of the $\mathrm{C} 3 \mathrm{H}$ and MAOA-K0 genotypes were lesioned at $\mathrm{P0}-\mathrm{P} 1, \mathrm{P2}-\mathrm{P3}$, or $\mathrm{P} 4-\mathrm{P} 5$. The effects on barrel emergence were evaluated with 5-HTT or $\mathrm{CO}$ staining at P9, P10, or P12. For each experimental group, cases were classified according to the degree of barrel segregation into three classes. Class I cases have a complete fusion of row C, class II cases have a partial fusion of row C, and class III cases have distinct barrel patterns in row C. Percentages of cases belonging to each class are provided for each experimental group.

ments within the cortex from sensory information coming from the whisker pad after P3 may rely on a somatotopic barrel-like organization fixed previously in the subcortical somatosensory nuclei at that time (Belford and Killackey, 1979). Along the same line, Boylan et al. (2001) showed that transection of the infraorbital nerve (ION) at P6 does not alter the emergence of barrels after a clorgyline treatment. Thus, barrel-like patterns and the relative space devoted to a lesioned or an unlesioned whisker could be imprinted in the principal trigeminal nucleus and in the ventrobasal thalamus by $\mathrm{P} 3$. This topographic information would thus be retained in these subcortical nuclei and would not require additional peripheral inputs for the actual remodeling of axons when the inhibitory action of serotonin on these processes is removed.

Our results also indicate that high concentrations of 5-HT do not prevent or modulate, in any visible manner, the effects of lesion-induced plasticity. In our model, the whisker row lesions at P1 and $\mathrm{P} 2$ are made in a context of raised levels of serotonin. Brain levels of 5-HT are nine times higher in the MAOA-KO mice compared with normal mice (Cases et al., 1995), and, when PCPA is administered at $\mathrm{P} 2$, it begins to effectively reduce $5-\mathrm{HT}$ levels by P3-P4. Thus, the plastic remodeling processes that conduct the afferents from the lesioned whisker to retract and conduct the afferents from neighboring unlesioned whiskers to expand do not appear to be controlled by serotonin. This suggests that glutamatergic neurotransmission is not required in this process because $5-\mathrm{HT}_{1 \mathrm{~B}}$ receptor activation reduces glutamatergic neurotransmission (Rhoades et al., 1994; Laurent et al., 2002). Glutamatergic activity was initially shown to be implicated in lesion-induced plasticity in the barrelfield by pharmacological experiments (Schlaggar et al., 1993). However, experiments using cortex-restricted NR1 (NMDA receptor 1)-KO mice showed no modification of the lesion-induced plasticity 
(Datwani et al., 2002). Similarly, NR2A-KO mice have a normal plasticity period (Lu et al., 2001).

It has been proposed that a molecular signal transmitted from the periphery could be important for somatotopic patterning and its plastic changes after lesion. Evidence in favor of this comes from experiments in which anterograde transport from the periphery was interrupted by vinblastine application to the ION, causing altered barrel development very similar to ION transection (Chiaia et al., 1996). However, the nature of this peripheral signal remains to be elucidated. Neurotrophic factors produced in the whisker pad, such as BDNF or neurotrophin 3 (Calia et al., 1998), have been proposed as possible candidates because their application on lesioned-whisker rows rescue the patterning of their corresponding barrel in the cortex.

In conclusion, our results indicate that excess 5-HT does not affect lesion-induced plasticity, although it profoundly affects barrel development, suggesting mechanistic differences between both processes. The end of lesion-induced plasticity may be determined in subcortical relays rather than in the cortex itself.

\section{References}

Belford GR, Killackey HP (1979) The development of vibrissae representation in subcortical trigeminal centers of the neonatal rat. J Comp Neurol 188:63-74.

Belford GR, Killackey HP (1980) The sensitive period in the development of the trigeminal system of the neonatal rat. J Comp Neurol 193:335-350.

Boylan CB, Kesterson KL, Bennett-Clarke CA, Chiaia NL, Rhoades RW (2001) Neither peripheral nerve input nor cortical NMDA receptor activity are necessary for recovery of a disrupted barrel pattern in rat somatosensory cortex. Brain Res Dev Brain Res 129:95-106.

Calia E, Persico AM, Baldi A, Keller F (1998) BDNF and NT-3 applied in the whisker pad reverse cortical changes after peripheral deafferentation in neonatal rats. Eur J Neurosci 10:3194-3200.

Cases O, Seif I, Grimsby J, Gaspar P, Chen K, Pournin S, Muller U, Aguet M, Babinet C, Shih JC, De Maeyer E (1995) Aggressive behavior and altered amounts of brain serotonin and norepinephrine in mice lacking MAOA. Science 268:1763-1766.

Cases O, Vitalis T, Seif I, De Maeyer E, Sotelo C, Gaspar P (1996) Lack of barrels in the somatosensory cortex of monoamine oxidase A-deficient mice: role of a serotonin excess during the critical period. Neuron 16:297-307.

Chiaia NL, Bennett-Clarke CA, Crissman RS, Zheng L, Chen M, Rhoades RW (1996) Effect of neonatal axoplasmic transport attenuation in the infraorbital nerve on vibrissae-related patterns in the rat's brainstem, thalamus and cortex. Eur J Neurosci 8:1601-1612.

Datwani A, Iwasato T, Itohara S, Erzurumlu RS (2002) Lesion-induced thalamocortical axonal plasticity in the $\mathrm{S} 1$ cortex is independent of NMDA receptor function in excitatory cortical neurons. J Neurosci 22:9171-9175.

Gaspar P, Cases O, Maroteaux L (2003) The developmental role of serotonin: news from mouse molecular genetics. Nat Rev Neurosci 4:1002-1012.

Jeanmonod D, Rice FL, Van der Loos H (1981) Mouse somatosensory cortex: alterations in the barrelfield following receptor injury at different early postnatal ages. Neuroscience 6:1503-1535.
Kidd FL, Coumis U, Collingridge GL, Crabtree JW, Isaac JT (2002) A presynaptic kainate receptor is involved in regulating the dynamic properties of thalamocortical synapses during development. Neuron 34:635-646.

Killackey HP, Leshin S (1975) The organization of specific thalamocortical projections to the posteromedial barrel subfield of the rat somatic sensory cortex. Brain Res 86:469-472.

Laurent A, Goaillard JM, Cases O, Lebrand C, Gaspar P, Ropert N (2002) Activity-dependent presynaptic effect of serotonin $1 \mathrm{~B}$ receptors on the somatosensory thalamocortical transmission in neonatal mice. J Neurosci 22:886-900.

Lebrand C, Cases O, Adelbrecht C, Doye A, Alvarez C, El Mestikawy S, Seif I, Gaspar P (1996) Transient uptake and storage of serotonin in developing thalamic neurons. Neuron 17:823-835.

Lu HC, Gonzalez E, Crair MC (2001) Barrel cortex critical period plasticity is independent of changes in NMDA receptor subunit composition. Neuron 32:619-634.

Nicol X, Ravary A, Bachy I, Muzerelle A, Gaspar P (2005) Spatiotemporal localisation of the calcium stimulated cyclase, AC1 and AC8, during mouse brain development. J Comp Neurol, in press.

Osterheld-Haas MC, Van der Loos H, Hornung JP (1994) Monoaminergic afferents to cortex modulate structural plasticity in the barrelfield of the mouse. Brain Res Dev Brain Res 77:189-202.

Rebsam A, Seif I, Gaspar P (2002) Refinement of thalamocortical arbors and emergence of barrel domains in the primary somatosensory cortex: a study of normal and monoamine oxidase a knock-out mice. J Neurosci 22:8541-8552.

Rhoades RW, Bennett-Clarke CA, Shi MY, Mooney RD (1994) Effects of 5-HT on thalamocortical synaptic transmission in the developing rat. J Neurophysiol 72:2438-2450.

Salichon N, Gaspar P, Upton AL, Picaud S, Hanoun N, Hamon M, De Maeyer E, Murphy DL, Mossner R, Lesch KP, Hen R, Seif I (2001) Excessive activation of serotonin (5-HT) $1 \mathrm{~B}$ receptors disrupts the formation of sensory maps in monoamine oxidase A and 5-HT transporter knock-out mice. J Neurosci 21:884-896.

Schlaggar BL, Fox K, O’Leary DD (1993) Postsynaptic control of plasticity in developing somatosensory cortex. Nature 364:623-626.

Senft SL, Woolsey TA (1991) Growth of thalamic afferents into mouse barrel cortex. Cereb Cortex 1:308-335.

Van der Loos H, Woolsey TA (1973) Somatosensory cortex: structural alteration following early injury to sense organs. Science 179:395-398.

Vongdokmai R (1980) Effect of protein malnutrition on development of mouse cortical barrels. J Comp Neurol 191:283-294.

Wong-Riley MT, Welt C (1980) Histochemical changes in cytochrome oxidase of cortical barrels after vibrissal removal in neonatal and adult mice. Proc Natl Acad Sci USA 77:2333-2337.

Woolsey TA, Van der Loos H (1970) The structural organization of layer V in the somatosensory region (SI) of the mouse cerebral cortex: the description of a cortical field composed of discrete cytoarchitectonic units. Brain Res 17:205-242.

Woolsey TA, Wann JR (1976) Areal changes in mouse cortical barrels following vibrissal damage at different postnatal ages. J Comp Neurol 170:53-66.

Woolsey TA, Dierker ML, Wann DF (1975) Mouse SmI cortex: qualitative and quantitative classification of golgi-impregnated barrel neurons. Proc Natl Acad Sci USA 72:2165-2169. 